\title{
Effects of structural irregularities on modulational instability phase matching in photonic crystal fibers
}

\author{
Bertrand Kibler, Cyril Billet, and John M. Dudley \\ Laboratoire d'Optique P. M. Duffieux, Université de Franche-Comté, 25030 Besançon, France
}

R. S. Windeler

OFS Laboratories, 700 Mountain Avenue, Murray Hill, New Jersey 07974

Guy Millot

Laboratoire de Physique de l'Université de Bourgogne, 21078 Dijon, France

\begin{abstract}
The effect of structural irregularities in photonic crystal fibers on scalar and vector modulational instability (MI) processes is studied by numerical simulations and experiments. For an anomalous-dispersion regime pump, variations in core ellipticity as small as $0.5 \%$ over length scales of the order of several meters are shown to have a negligible effect on scalar MI, yet they completely suppress vector MI. In contrast, for a normal-dispersion regime pump, vector MI is shown to be robust against such fluctuations.
\end{abstract}

The ability to engineer the dispersion and nonlinearity of photonic crystal fibers (PCFs) has led to the observation of a number of novel nonlinear effects in optical fibers. ${ }^{1-3}$ Modulational instability (MI) is a particular nonlinear process that has been the subject of recent study in PCFs, leading to the discovery of a new scalar MI window in the normal-dispersion regime $^{4}$ that is due to higher-order dispersion phase matching. ${ }^{5,6}$ The design freedom of PCFs allows unusual dispersion to be combined with engineered birefringence, and preliminary studies of polarizationdependent vector MI in PCFs have also been reported. ${ }^{7}$ Recent theoretical work has also treated related processes in small-core fibers with dispersion profiles similar to those of PCFs. ${ }^{8}$

MI is associated with exponential gain for signals at frequencies symmetric about an injected continuouswave pump and has important applications in parametric frequency conversion and in the generation of ultrashort pulse trains. ${ }^{9}$ MI gain depends on phase-matching conditions that involve fiber nonlinearity, group-velocity dispersion (GVD), and, for vector $\mathrm{MI}$, the group-velocity mismatch (GVM) between orthogonal polarizations. These parameters in a solid core PCF depend strongly on the air hole distribution about the core, and irregularities in the PCF structure would be expected to affect the MI gain significantly. In this Letter we present a numerical and experimental study of these effects, including the sensitivity of both scalar and vector MI processes to structural irregularities along the length of an ellipticalcore birefringent PCF. Beam propagation method calculations allow GVD and GVM variations to be determined directly from PCF structural fluctuations, and MI gain calculations and nonlinear Schrödinger equation simulations are used to examine the conse- quences for both scalar and vector instabilities. Our major result is that variations in core ellipticity as small as $0.5 \%$ over several meters have significantly different effects on scalar and vector MI processes in the anomalous- and normal-dispersion regimes. In particular, for anomalous-dispersion regime pumping, structural irregularities are shown to have little effect on scalar MI but to completely suppress vector MI processes that depend on polarization coupling.

We consider $3.9 \mathrm{~m}$ of OFS Laboratories PCF based on a hexagonal arrangement of circular air holes surrounding a central solid elliptical core (see Ref. 1 for an electron micrograph). The zero-dispersion wavelength is $\sim 760 \mathrm{~nm}$, and the birefringence is $\Delta n \sim 10^{-3}$. The hexagonal cladding design is standard for highly nonlinear PCF and is usually specified in terms of air hole diameter $\phi$ and the center-to-center spacing between adjacent holes $\Lambda .^{5}$ One can model the elliptical core by inscribing the ring of air holes immediately surrounding the core in an ellipse with semimajor and semiminor axes $(1+\epsilon) \Lambda$ and $\Lambda$, respectively. ${ }^{7}$

Assuming identical GVD and equal pumping along each axis of a highly birefringent fiber, the expected MI gain ${ }^{9}$ is $g=2 \operatorname{Im}(K)$. Here, the wave vector $K$ is obtained from the dispersion relation $\left[(K-b)^{2}-\right.$ $H]\left[(K+b)^{2}-H\right]=C_{x}{ }^{2}$, where $\Omega$ is the frequency offset from the pump and $b=\left(\beta_{1 x}-\beta_{1 y}\right) \Omega / 2$ takes into account the GVM. $\quad H=\beta_{2} \Omega^{2}\left(\beta_{2} \Omega^{2} / 4+\gamma P\right)$, and the cross-phase modulation parameter $C_{x}=(2 / 3) \beta_{2} \gamma P \Omega^{2}$. The fiber GVD and GVM were determined for wavelengths in both the anomalous- $(\lambda=1064 \mathrm{~nm})$ and the normal- $(\lambda=624.5 \mathrm{~nm})$ dispersion regimes by use of imaginary distance vector beam propagation method calculations. $^{10}$ Parameters $\phi=1.4 \mu \mathrm{m}, \Lambda=1.6 \mu \mathrm{m}$, and $\epsilon=0.05$ yielded values of GVD and GVM in excellent agreement with experiments. We used a 
convergence study to test the calculation fidelity by methodically reducing the computational resolution to determine optimal values that maximized accuracy and minimized computation speed. Transverse and longitudinal resolutions of $\lambda / 20$ were found to yield consistent results.

The calculated GVD for the polarization eigenaxes differed by only $2 \%$, consistent with the assumption of the linearized theory. We also used calculations to determine nonlinear parameter $\gamma$. The values obtained at $1064 \mathrm{~nm}$ were $\left|\beta_{1 x}-\beta_{1 y}\right|=3.25 \mathrm{ps} \mathrm{m}^{-1}$, average $\beta_{2}=-77.5 \mathrm{ps}^{2} \mathrm{~km}^{-1}$, and $\gamma=76 \mathrm{~W}^{-1} \mathrm{~km}^{-1}$. At $624.5 \mathrm{~nm}$ the values obtained were $\left|\beta_{1 x}-\beta_{1 y}\right|=$ $0.947 \mathrm{ps} \mathrm{m}^{-1}$, average $\beta_{2}=26.9 \mathrm{ps}^{2} \mathrm{~km}^{-1}$, and $\gamma=$ $149 \mathrm{~W}^{-1} \mathrm{~km}^{-1}$. We consider equal pump powers on each axis of $P=27.5 \mathrm{~W}$ at $1064 \mathrm{~nm}$ and $P=45 \mathrm{~W}$ at $624.5 \mathrm{~nm}$ (corresponding to our experiments below).

Figure 1 shows the gain curves calculated for these parameters. For an anomalous-dispersion regime pump, Fig. 1(a) shows that both scalar and vector MI are expected. The broad scalar MI gain peak grows from the pump $(\Omega=0)$, with a maximum near $\Omega_{s} / 2 \pi=1 / 2 \pi\left(2 \gamma P /\left|\beta_{2}\right|\right)^{1 / 2}=1.2 \mathrm{THz}$. The narrow vector MI peak is displaced near $\Omega_{v} / 2 \pi=1 / 2 \pi\left|\beta_{1 x}-\beta_{1 y}\right| /\left|\beta_{2}\right|=6.7 \mathrm{THz}$. For the normal-dispersion regime pump, Fig. 1(b) shows the vector gain peak centered on $\Omega_{v} / 2 \pi=3.7 \mathrm{THz}$. Complementary nonlinear Schrödinger equation simulations were also carried out for pump and fiber parameters as above, with Gaussian input pulses of durations $0.8 \mathrm{~ns}$ at $1064 \mathrm{~nm}$ and $1 \mathrm{~ns}$ at $624.5 \mathrm{~nm}$ (also corresponding to the experiment). The bottom curves in Fig. 1 present these results, clearly showing the growth of distinct scalar and vector MI sidebands in each case.

The positions of the MI gain peaks are, however, strongly dependent on the fiber GVM and GVD, and any longitudinal variations in these parameters would lead to reduced accumulated gain over the fiber length. In this context, we note that such variations have been extensively observed in standard fiber ${ }^{11}$ and were previously inferred for this PCF from differences in the zero-dispersion wavelength between fiber segments. ${ }^{12}$ Such variation can arise from complex changes in hole size and position along the fiber, but for purposes of studying the effect on MI we use a simplified model that involves only the longitudinal variation in the ellipticity parameter $\epsilon$ about its mean of 0.05. Based on electron micrograph images at different points ( $\sim 1-\mathrm{m}$ separation), we estimated an upper bound of $5 \%$ variation in ellipticity. Within this constraint, we used beam propagation method calculations to quantify the corresponding effect on GVD and GVM. The calculations showed that the GVM is particularly sensitive to structural nonuniformity, even with levels of ellipticity variation as low as $0.5 \%$. For example, a $0.5 \%$ variation in $\epsilon$ gave GVM variations over the range $2.9-3.6 \mathrm{ps} \mathrm{m}^{-1}$ ( $\sim 10 \%$ about the mean), whereas the corresponding variation in the average GVD (absolute value) was $76.5-78.5 \mathrm{ps}^{2} \mathrm{~km}^{-1}(\sim 1 \%$ about the mean). Note that the magnitudes of these parameter fluctuations that we obtained are comparable with those seen in standard fiber ${ }^{11}$ and with those used in recent studies of scalar supercontinuum generation in PCF. ${ }^{13}$

We can conveniently examine the effects of these longitudinal fluctuations by assuming a $0.5 \%$ periodic variation in $\epsilon$ over a characteristic length of $2 \mathrm{~m}$ (consistent with fluctuation length scales of standard fibers ${ }^{11}$ ). To illustrate the consequences for the MI gain, in Fig. 2 we show again the MI gain curves but recalculated locally at different points along the fiber. In the anomalous-dispersion regime, Fig. 2(a) reveals a minimal effect on the position of the scalar MI gain peak, yet it shows a significant variation in the position of the vector MI gain peak. In the normaldispersion regime, Fig. 2(b) shows some variation in the vector MI gain curve, although the oscillation is less apparent because of the larger intrinsic MI gain bandwidth in this case.

(a) Anomalous Dispersion Pump

(b) Normal Dispersion Pump
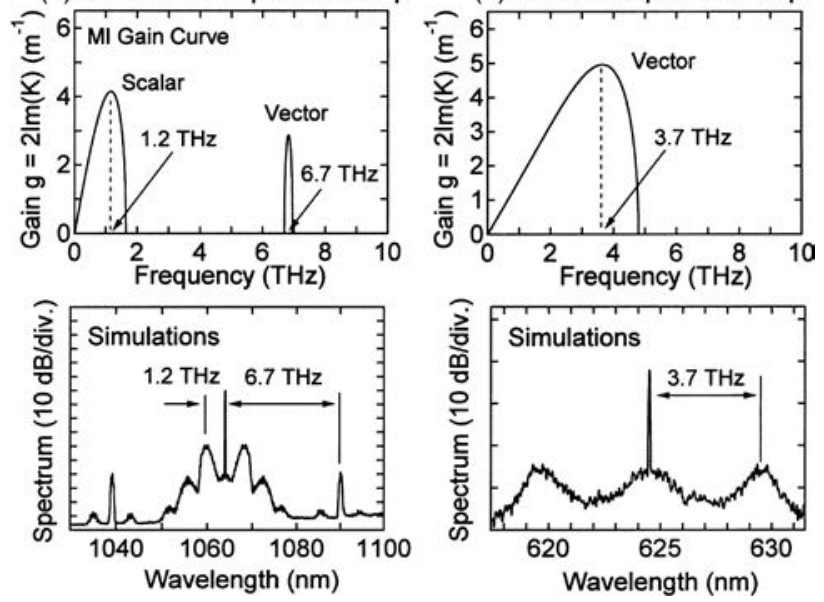

Fig. 1. Top, calculated MI gain curves and bottom, corresponding simulated output spectra for anomalous- and normal-dispersion regime pumps.

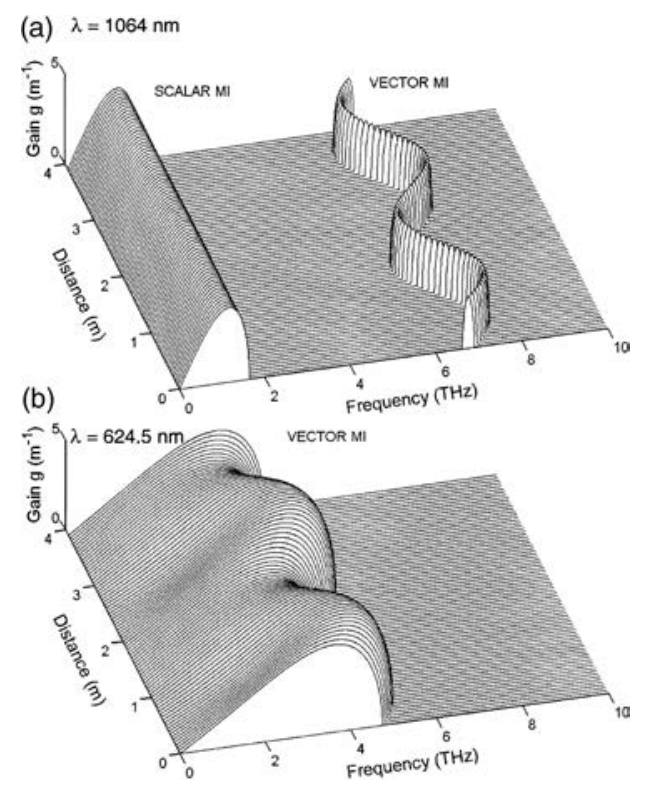

Fig. 2. Calculated MI gain curves assuming a periodic variation in core ellipticity of $0.5 \%$ for (a) anomalous- and (b) normal-dispersion regime pumps. 
(a) Anomalous Dispersion Pump
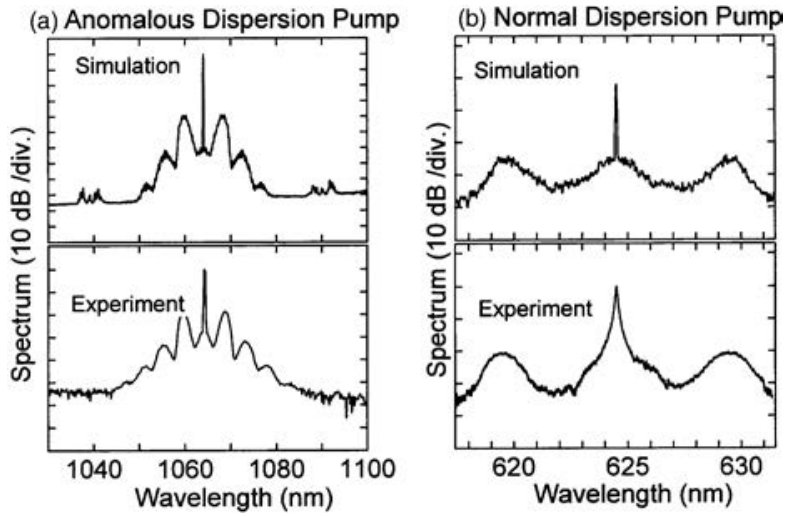

Fig. 3. Top, simulated and bottom, experimental output spectra after $3.9 \mathrm{~m}$ of PCF propagation with a pump polarized at $45^{\circ}$ to the principal axes for anomalous- and normaldispersion regime pumping.

For our parameters, the MI gain occurs on a length scale of $g^{-1} \approx 0.2 \mathrm{~m}$, which is much less than the length scale of $2 \mathrm{~m}$ that describes the longitudinal fluctuations. It would thus be expected that the oscillations seen in Fig. 2 would significantly modify the accumulated gain. However, Fig. 2 also suggests differences between the anomalous- and the normaldispersion regimes. In the anomalous-dispersion regime the robustness of scalar MI to structural irregularities would be expected to lead to exponential gain for this process. Yet, because the variation in the position of the vector MI peak exceeds its intrinsic peak width, the gain of vector MI would be reduced. In contrast, for the normal-dispersion regime, the greater intrinsic width of the vector MI peak ensures that, despite oscillations in position, there always remains a frequency range over which gain is present, so significant vector $\mathrm{MI}$ in this pumping regime would be expected.

This interpretation was confirmed by simulations that explicitly include the longitudinal GVD and GVM variations. Figure 3(a) shows results for the anomalous-dispersion regime. In contrast with the simulations with constant parameters shown in Fig. 1(a) (where the amplitude of the vector MI peak was comparable with the second-order scalar MI peak), the vector MI peak in the presence of varying GVM is almost completely suppressed. The scalar sidebands are still clearly observed, however. For the normal-dispersion regime, Fig. 3(b) shows clear vector MI sidebands even with varying GVM. We note also that, although these results were obtained with the assumption of a smooth periodic variation in the PCF dimensions, we have verified that simulations that use other models for the longitudinal parameter variation yield comparable results. ${ }^{11,13}$

These MI processes were also studied experimentally. At $1064 \mathrm{~nm}, 0.8-\mathrm{ns}$ pulses from a microchip laser were injected equally along the polarization eigenaxes of a 3.9-m PCF with parameters as above. The output spectrum was measured for the different polarization states by an optical spectrum analyzer with dynamic range sufficient to permit nearly four orders of scalar MI to be observed. The bottom curve in Fig. 3(a) shows the results. Although, in the absence of structural nonuniformity the vector MI peak would be expected to have an amplitude comparable with the second-order scalar MI peak (see Fig. 1), no experimental evidence of vector MI is seen. We interpret the absence of the vector MI peak as confirming the suppression of vector MI caused by variation in the longitudinal GVM. In contrast, when 1-ns pulses were used at $624.5 \mathrm{~nm}$ (in the same PCF), the bottom curve of Fig. 3(b) shows that clear vector MI sidebands were observed. This result confirms the robustness of vector MI in the normal-dispersion regime to GVM variations.

The important conclusion to be drawn from our results is that irregularities in a PCF structure at a level that has negligible consequences for scalar nonlinear effects can nonetheless strongly influence polarizationdependent vector nonlinear processes. Although we have considered only modulational instability, a similar conclusion would be expected to hold for other nonlinear processes in PCF when they involve coupling between orthogonal polarizations. Our results have important consequences for the design of PCF-based devices that rely on polarization-dependent nonlinear effects, as it implies the need for stringent design and manufacturing tolerances to prevent longitudinal GVM fluctuations in the GVM. Our results also indicate, however, that the introduction of controlled GVM variations will be advantageous when the suppression of polarization-dependent nonlinearities is desirable.

J. M. Dudley's e-mail address is john.dudley@ univ-fcomte.fr.

\section{References}

1. J. K. Ranka, R. S. Windeler, and A. J. Stentz, Opt. Lett. 25, 25 (2000).

2. W. J. Wadsworth, J. C. Knight, A. Ortigosa-Blanch, J. Arriaga, E. Silvestre, and P. St. J. Russell, Electron. Lett. 36, 53 (2000).

3. D. Skryabin, F. Luan, J. C. Knight, and P. St. J. Russell, Science 301, 1705 (2003).

4. J. D. Harvey, R. Leonhardt, S. Coen, G. K. L. Wong, J. C. Knight, W. J. Wadsworth, and P. St. J. Russell, Opt. Lett. 28, 2225 (2003).

5. W. H. Reeves, D. V. Skryabin, F. Biancalana, J. C. Knight, P. St. J. Russell, F. G. Omenetto, A. Efimov, and A. J. Taylor, Nature 424, 511 (2003).

6. S. Pitois and G. Millot, Opt. Commun. 226, 415 (2003).

7. G. Millot, A. Sauter, J. M. Dudley, L. Provino, and R. S. Windeler, Opt. Lett. 27, 695 (2002).

8. F. Biancalana and D. Skyrabin, J. Opt. A 6, 301 (2004).

9. G. P. Agrawal, Nonlinear Fiber Optics, 3rd ed. (Academic, New York, 2001).

10. BandSOLVE 1.3 (RSoft Design Group, New York, 2003).

11. M. Karlsson, J. Opt. Soc. Am. B 15, 2269 (1998).

12. J. E. Sharping, M. Fiorentino, A. Coker, P. Kumar, and R. S. Windeler, Opt. Lett. 26, 1048 (2001).

13. N. I. Nikolov, T. Sorenson, O. Bang, and A. Bjarklev, J. Opt. Soc. Am. B 11, 2329 (2003). 